\title{
Errata: Computing the solid angle subtended by a planar figure
}

John S. Asvestas

U.S. Navy Naval Air Systems Command

Radar and Antenna Systems Division

B 2187, S 2190

Patuxent River, Maryland 20670-1906

E-mail: john.asvestas@navy.mil

[DOI: 10.1117/1.3582189]

This article [Opt. Eng. 33(12), 4055-4059 (1994)] was published in December 1994. We correct two mathematical expressions.

In the original paper, a negative sign is missing in Eq. (6). The correct expression is $\hat{\xi}=\hat{\eta} \times \hat{\zeta}=-\frac{\hat{R}_{n} \times\left(\hat{R}_{n} \times \hat{R}_{n+1}\right)}{\left|\hat{R}_{n} \times \hat{R}_{n+1}\right|}$

The same mistake appears in Eq. (16). The correct expression is

$I_{n}=2 \arctan \left[\frac{\frac{\hat{q} \cdot\left(\hat{R}_{n} \times \hat{R}_{n+1}\right)}{\left|\hat{R}_{n} \times \hat{R}_{n+1}\right|}\left(\frac{1-\hat{R}_{n} \cdot \hat{R}_{n+1}}{1+\hat{R}_{n} \cdot \hat{R}_{n+1}}\right)^{1 / 2}}{1-\hat{q} \cdot \hat{R}_{n}+\frac{\hat{q} \cdot\left[\hat{R}_{n} \times\left(\hat{R}_{n} \times \hat{R}_{n+1}\right)\right]}{\left|\hat{R}_{n} \times \hat{R}_{n+1}\right|}\left(\frac{1-\hat{R}_{n} \cdot \hat{R}_{n+1}}{1+\hat{R}_{n} \cdot \hat{R}_{n+1}}\right)^{1 / 2}}\right]$.

All the results presented in the original paper are correct.

In numerical implementations* the atan 2 function should be used for the arctangent.

*http://en.wikipedia.org/wiki/Atan2. 